\title{
COMMENTAIRES DE LA SOCIÉTÉ FRANCCAISE
DE PHYSIQUE
}

\author{
Charles-Henri de Novion
}

Le rapport "La Métallurgie, science et ingénierie » présente de manière très claire et convaincante l'importance de l'industrie métallurgique pour la France, le caractère spécifique de la science qui y est associée, et les défis auxquels est confrontée actuellement cette discipline. Le rapport principal est accompagné de 25 annexes, toutes intéressantes, et dont certaines sont tout à fait remarquables, comme l'annexe 3 sur la métallurgie fondamentale et la métallurgie numérique, ou l'annexe XXV sur l'enseignement de la métallurgie.

Un des points principaux (et particulièrement inquiétant) qui se dégage du rapport est la désaffection des jeunes pour la métallurgie, ce que traduit en particulier la phrase : "La Métallurgie n'apparaît pas comme une matière scientifique de pointe ni comme un besoin pour la société ». Ce constat dépasse le cas des étudiants. On le trouve dans beaucoup de milieux de la recherche, en particulier en physique. À cela est liée l'absence (presque) totale de la science et de l'ingénierie métallurgiques dans les médias de grande diffusion scientifique : La Recherche, Pour la Science, etc. Sans doute même dans les faits marquants et communiqués de presse des grands organismes comme le CNRS et le CEA. Il semble donc qu'une action vigoureuse de "popularisation " des percées scientifiques, technologiques et industrielles, en métallurgie, est absolument nécessaire. II faut aussi prendre appui sur le caractère interdisciplinaire de la science métallurgique, et sur le fait que des concepts qui y ont été développés se retrouvent dans d'autres disciplines (par exemple, la germination-croissance des galaxies à partir des fluctuations du fond diffus cosmologique).

En ce qui concerne le rapport principal :

1. L'introduction est tout à fait remarquable, posant très bien le problème.

2. Le "résumé et recommandations", très clair, est une bonne synthèse du rapport. Peut-être, toutefois, présente-t-il le défaut de parler simplement de "restauration » de la discipline Métallurgie. Le point important, bien expliqué ensuite dans l'introduction, que cela ne peut pas être une restauration de la métallurgie d'avant-hier, qu'il ne faut plus faire la course 
à la production, mais choisir des créneaux d'excellence, aurait dû y être mentionné et même souligné.

3. L'équilibre entre les trois chapitres n'est pas excellent. II faudrait donner plus d'importance au chapitre 3 (" La Métallurgie : recherche et enseignement ", qui ne fait actuellement que 5 pages), par rapport au chapitre 1 et surtout au chapitre 2 (28 pages) qui, par moments, rentrent trop dans les détails.

4. On ne sait pas très bien à quel public s'adresse ce rapport, mais il me semble qu' une version courte (introduction + environ 10 à 15 pages) serait très utile.

5. On peut noter une grande hétérogénéité des recommandations, ce qui est un peu gênant. Certaines sont des constats, d'autres des actions à entreprendre. Cela doit dépendre aussi du rédacteur de la partie concernée. Par exemple, dans le chapitre 1, les recommandations de la section 1 ("Métallurgie physique ») sont de caractère général et claires pour tout lecteur; en revanche, celles de la section 2 ("Métallurgie et mécanique ») sont très techniques, et peut-être trop spécialisées au niveau de ce rapport de synthèse.

6. Quelques sujets importants auraient dû être plus discutés, en particulier les contrôles non destructifs et la corrosion. On aurait pu mentionner le problème très important de la métallurgie du silicium pour le photovoltaïque (une métallurgie tout à fait classique par réduction d'oxyde), ainsi que celui des fils de cuivre pour applications électriques (dont la qualité mécanique est très sensible à des taux infimes d'impuretés, $<\mathrm{ppm}$ ). 